\title{
Caracterisation Physico-Chimique Des Eaux Du Barrage Foum El-Khanga (Region De Souk-Ahras, Algerie)
}

\author{
Amel Allalgua, Dr. \\ Nouha Kaouachi, MCA \\ Chahinez, Boualeg, MCA \\ Adel Ayari, MAA
}

Univ Md Chérif Messadia Souk Ahras, Fac. SNV, Laboratoire des

Ecosystèmes Aquatiques et Terrestres, Bp 1553, Route d'Annaba, Souk

Ahras, Algerie.

Mourad Bensouileh, Prof.

Univ Badji Mokhtar Annaba, Fac. Sci, Laboratoire d'Ecobiologie des

Milieux Marins et littoraux, BP12 Annaba, Algerie.

doi: 10.19044/esj.2017.v13n12p258 URL:http://dx.doi.org/10.19044/esj.2017.v13n12p258

\begin{abstract}
The objective of our work is to assess the quality of the dam Foum El-Khanga (east of Algéria) monthly during the year 2014. This study planned to make a physico-chemical characterization to highlight several parameters which come into play the determination of the quality of this water such as: temperature; $\mathrm{pH}$; conductivity; turbidity; dissolved oxygen; title hydrotimetric; calcium; magnesium; title alcalimetric complet; nitrate; nitrite; ammoniacal nitrogen; sulfate; chloride; bicarbonate; phosphate; iron; biological oxygen demand (BOD) and chemical oxygen demands (COD). The results of physico-chemical analysis show that the water of this dam is characterized by a significant mineralization as indicated by high values of electrical conductivity and chloride.

The results of principal component analysis (PCA) clearly showed a stronger relation between the hot season and temperature. However, the cold season was strongly correlated with mineralization and pollution parameters.
\end{abstract}

Keywords: Foum El-Khanga dam, east of Algéria, Physico-chemical parameters, Irrigation water, PCA. 


\section{Résumé}

L'objectif de notre travail est d'évaluer la qualité des eaux du barrage Foum El-Khanga (est de l'Algérie) mensuellement durant l'année 2014. Cette étude a envisagé de faire une caractérisation physico-chimique pour mettre en évidence plusieurs paramètres qui entrent en jeu dans la détermination de la qualité de cette eau tel que : la température ; le $\mathrm{pH}$; la conductivité ; la turbidité ; l'oxygène dissous ; le titre hydrotimétrique ; le calcium ; le magnésium ; le titre alcalimétrique complet ; le nitrate ; le nitrite ; l'azote ammoniacal ; le sulfate ; le chlorure ; le bicarbonate ; le phosphate ; le fer; la demande biologique en oxygène $\left(\mathrm{DBO}_{5}\right)$ et la demande chimique en oxygène $(\mathrm{DCO})$.

Les résultats des analyses physico-chimiques montrent que les eaux de ce barrage sont caractérisées par une minéralisation naturelle importante comme l'indique les valeurs élevées de la conductivité électrique et des chlorures.

Les résultats de l'analyse en composantes principales (ACP) ont clairement montré une forte corrélation entre la saison chaude et la température. En revanche, la saison froide est fortement corrélée avec les paramètres de minéralisation et de pollution.

Mots-clés : Barrage Foum El-Khanga, est de l'Algérie, Paramètres physicochimiques, Eau d'irrigation, ACP.

\section{Introduction}

En Algérie, les eaux de surface sont de plus en plus utilisées ces dernières années pour les besoins de l'agriculture, de l'alimentation des populations et de l'industrie. Des investissements considérables ont été consacrés à la construction des barrages, notamment dans l'Est du pays. Cependant, ces eaux de surface sont vulnérables face aux diverses pollutions et sont souvent de qualité médiocre. Elles peuvent contenir des quantités non négligeables en matières organiques naturelles telles que les substances humiques mais aussi des composés organiques issus de divers rejets polluants ou de pratiques agricoles intensives (Achour, 2001). En Algérie, certaines études portant sur la qualité physico-chimique des eaux des barrages, nous citons : Halimi (2008) dans le bassin versant d'oued Cherf; Harrat et Achour (2010) dans les deux barrages Mexa et Chefia; Merabet (2010) dans le barrage de Beni Haroun; Harrat et Achour (2011) dans le barrage Zit El-Emba; Ouahchia et al. (2015) dans le lac du barrage Boukourdane. Ils ont pu estimer leur potabilité, leur aptitude à l'irrigation, et par conséquent, leur impact sur la santé humaine et environnementale (Kahoul et Touhami, 2014). 
Notre préoccupation a pour objectif d'étudier pour la première fois la qualité physico-chimique des eaux du barrage Foum El-Khanga $\left(152\right.$ millions $\left.\mathrm{m}^{3}\right)$ situé dans la Wilaya de Souk-Ahras (Nord-Est de l'Algérie) et dirigé vers l'irrigation agricole.

\section{Matériel et méthodes}

\section{Zone d'étude}

Le barrage Foum El-Khanga est construit en 1994, s'étale sur une superficie de $1735 \mathrm{~km}^{2}$ avec un périmètre de $190 \mathrm{Km}$ (Figure 1). Il est situé dans la partie Nord-Est du territoire algérien sur l'Oued Cherf au lieu dit Foum El-Khanga à mi-distance, entre Sedrata et Ksar Sbahi $(20 \mathrm{~km}$ environ de part et d'autre), il constitue la région limitrophe de trois wilaya : SoukAhras ; Guelma et Oum El Bouaghi et regroupe 7 daïras et 16 communes.

Figure 1 : Situation géographique du barrage Foum El-Khanga.

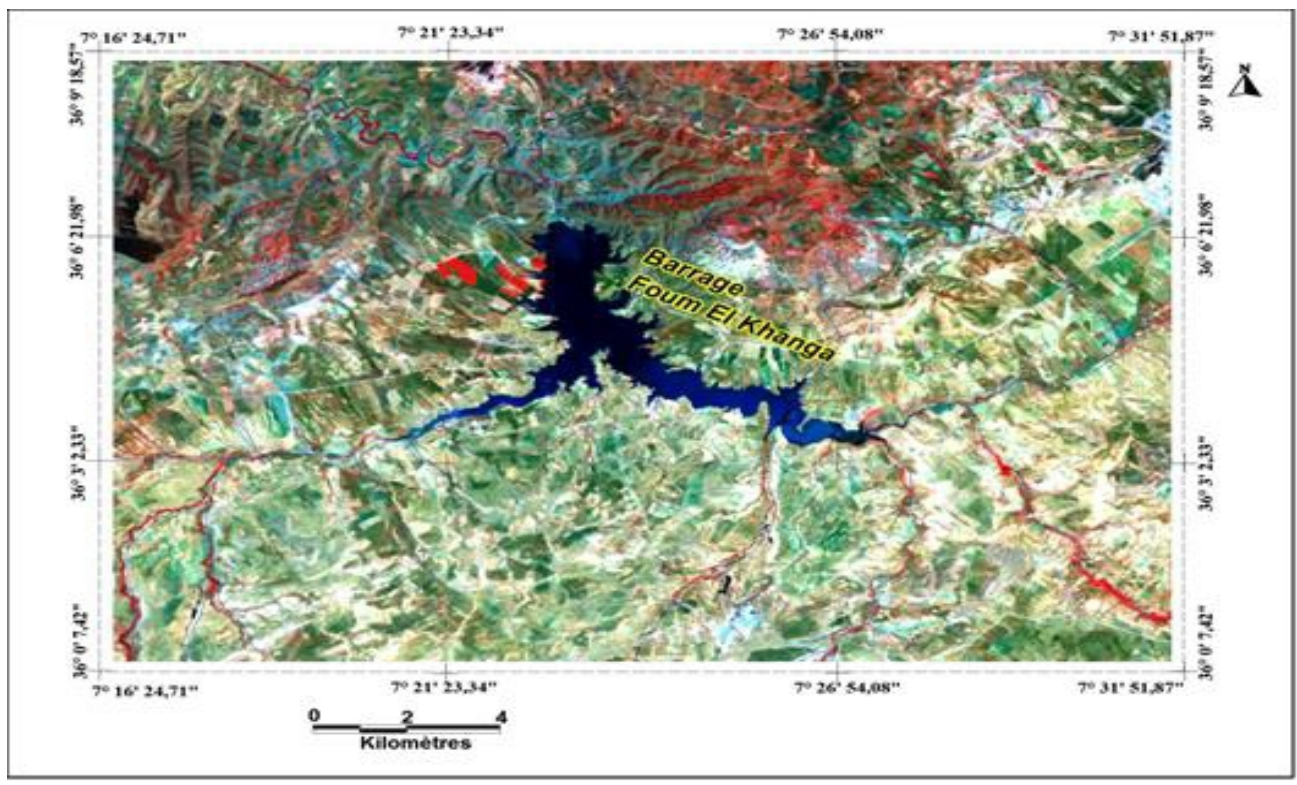

\section{Méthode d'étude}

Pour la réalisation de cette étude, nous avons effectué 12 prélèvements mensuelles tous le long de l'année 2014 dans des flacons en polyéthylène de capacité d'un litre, à une profondeur de $3 \mathrm{~m}$ d'après la digue de ce barrage. Tous flacons d'échantillonnage sont clairement identifiés et accompagnés d'informations suffisantes concernant la nature de l'échantillon et les raisons pour lesquelles l'examen est effectué et transporté dans une glacière avec un délai maximum de 8 heures avant l'analyse.

Les caractéristiques physico-chimiques ont été établies selon les normes AFNOR (1985) et Rodier (1984), celles-ci sont consignées dans le tableau 1. 
Tableau 1 : Caractéristiques physico-chimiques étudiées

\begin{tabular}{|c|c|c|}
\hline lieu d'analyse & paramètres effectué & nature de la méthode \\
\hline $\begin{array}{c}\text { Analyse in situ } \\
\text { (paramètres } \\
\text { physiques) }\end{array}$ & $\begin{array}{c}\mathrm{pH} \text {, température, conductivité } \\
\text { électrique, turbidité et } \\
\text { l'oxygène dissous }\end{array}$ & Méthode électro-métrique avec \\
électrode combinée \\
\hline Analyse in vitro & Azote ammoniacal & \\
(paramètres & Dureté $\left(\mathrm{Ca}^{+2}+\mathrm{Mg}^{+2}\right)$ & Méthode Spectrométrique U.V Visible \\
chimiques) & TAC et l'Alcalinité & Méthode Tetrimetrique à l'EDTA \\
& Sulfates & Méthode Tetrimetrique à HCl \\
& Fer & Méthode Spectrométrique \\
& Chlorures & Méthode Spectrométrique à la phenanthroline-1,10 \\
& Nitrites & Méthode Tetrimetrique à Ag NO $\mathrm{NO}_{3}$ \\
& Nitrates & Méthode Spectrométrique \\
& Phosphates & Méthode au Salicylate de Sodium \\
& DBO & Méthode Spectrométrique \\
& DCO & DBO-mètre \\
& distroussium \\
\hline
\end{tabular}

Pour déterminer la distribution des paramètres étudiés selon les mois d'étude, nous avons utilisé la méthode explicative par l'analyse en composantes principales (ACP). Cette méthode est largement utilisée pour interpréter les données hydro-chimiques (Chaouki et al., 2015 ; Lakhili et al., 2015), en utilisant le logiciel STATISTICA (StatSoft, version 10) pour WINDOWS.

\section{Résultats et discussion}

\section{Analyse physico-chimiques}

\section{Température d'eau}

Les valeurs de la température des 12 mois de l'année 2014 sont très variables, elles oscillent entre $7,2^{\circ} \mathrm{C}$ (mois de décembre) et $30^{\circ} \mathrm{C}$ au maximum (mois d'août), avec une moyenne de $16,37^{\circ} \mathrm{C}$ (Figure 2), elles sont voisines des températures ambiantes. Ces résultats restent inférieurs à ceux rapportés par Mehanned et al. (2014) au niveau du barrage Sidi Chahed (température moyenne varient entre $22,45^{\circ} \mathrm{C}$ à $\mathrm{Si}$ et $23,88^{\circ} \mathrm{C}$ ).

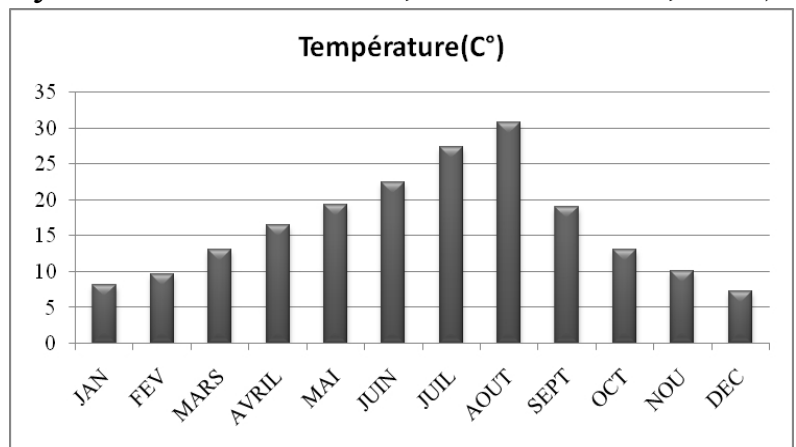

Figure 2: Variation temporelle de la température. 


\section{Potentiel d'hydrogène}

Les valeurs du $\mathrm{pH}$ enregistrées durant la période d'étude sont au voisinage de la neutralité dans les deux mois avril et mai avec un caractère plus ou moins alcalin durant le reste de l'année (Figure 3) et un maximum noté au mois d'août $(8,6)$. L'alcalinité de notre barrage est importante par rapport au barrage Hassan II qui ont varié globalement entre 7,06 et 7,53 (Chahboune et al., 2013).

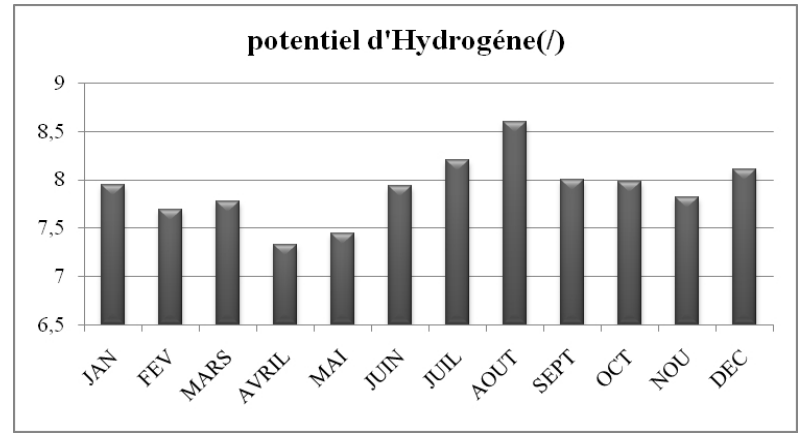

Figure 3 : Variation temporelle du potentielle d'hydrogène.

\section{Conductivité électrique}

La mesure de la conductivité permet d'évaluer rapidement mais très approximativement la minéralisation globale de l'eau et d'en suivre l'évolution (Rodier et al., 2009).

Dans notre zone d'étude, toutes les valeurs mesurées de la conductivité indiquent une minéralisation élevée car elles sont toutes globalement supérieures à $1500 \mu \mathrm{s} / \mathrm{cm}$ (Figure 4). Cette minéralisation importante traduit une forte teneur des cations et anions dans cette eau due probablement au drainage des terrains triasiques très riches en sels et en gypse (la nature géologique des couches traversées). Les valeurs obtenues s'avèrent largement inférieures à celles marquées par Bouderka et al. (2016) au niveau des eaux souterraines de la nappe du Gharb (oscillent entre 770 et $9890 \mu \mathrm{s} / \mathrm{cm})$.

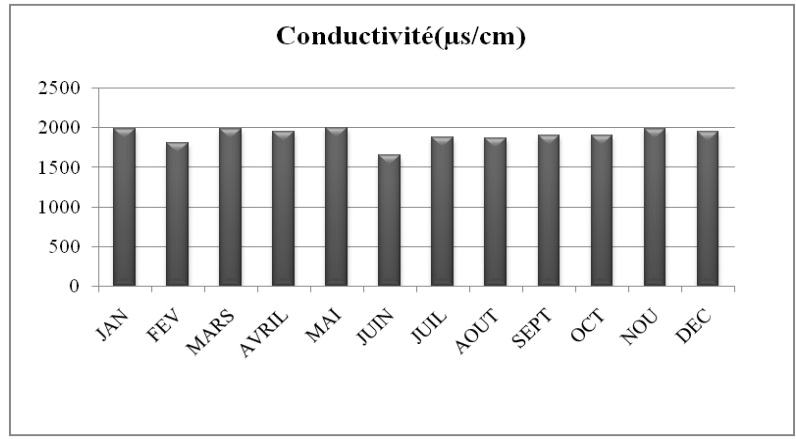

Figure 4 : Variation temporelle de la conductivité électrique. 


\section{Oxygène dissous}

L'oxygène est l'un des paramètres particulièrement utiles pour l'eau et constitue un excellent indicateur de sa qualité. C'est un des paramètres les plus sensibles à la pollution (Makhoukh et al., 2011).

Les valeurs obtenues de ce paramètre montrent une fluctuation allant de $5,12 \mathrm{mg} / \mathrm{l}$ (mois de juin) à $10 \mathrm{mg} / \mathrm{l}$ (mois de janvier), avec une moyenne de 7,70 mg/l (Figure 5). Les faibles teneurs en oxygène dissous sont observées durant les mois chauds ; car une eau froide contient une plus grande quantité d'oxygène dissous qu'une eau de température élevée (Hébert et Légaré, 2000).

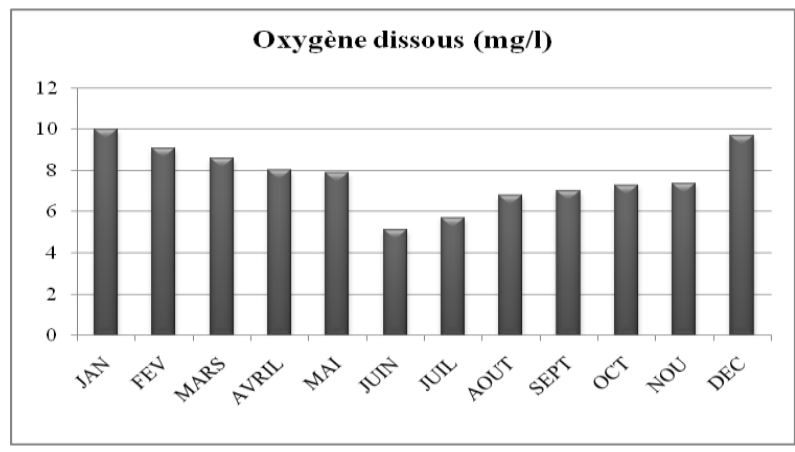

Figure 5 : Variation temporelle de l'oxygène dissous.

\section{Turbidité}

La turbidité résulte la présence des matières en suspension dans l'eau (débris organiques, argiles, organismes microscopiques...). L'appréciation de l'abondance de ces matières mesure son degré de turbidité (Jemali et Kefati, 2002).

Le suivi de ce paramètre donne des valeurs situant entre 4,6 NTU et 6,82 NTU, avec une moyenne de 5,83 NTU (Figure 6). Durant la saison sèche, la turbidité est faible par rapport à la saison pluvieuse où l'apport des eaux de ruissellement augmente la turbidité de l'eau (Ahonon, 2011).

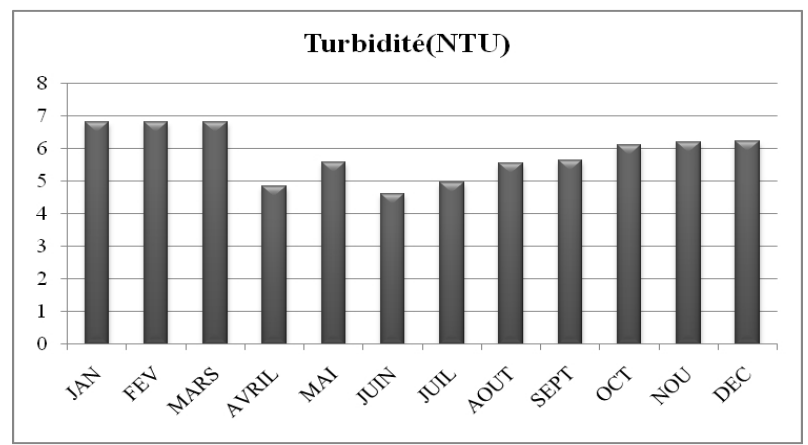

Figure 6 : Variation temporelle de la turbidité. 


\section{Dureté totale}

La dureté de l'eau se réduit à sa concentration en ions calcium et magnésium (Ghazali et Zaid, 2012). Dans les eaux étudiées (Figure 7), ce paramètre présente une grande variation, elle se diminue en période sèche et augmente en période pluvieuse, le taux le plus faible est enregistré au mois de juin $\left(22,1 \mathrm{~F}^{\circ}\right)$ et le taux le plus élevé est marqué au mois de février $(51,7$ $\mathrm{F}^{\circ}$ ). Cette variation serait liée à la nature lithologique de la formation aquifère et en particulier à sa composition en magnésium et en calcium (Derwich et al., 2010).

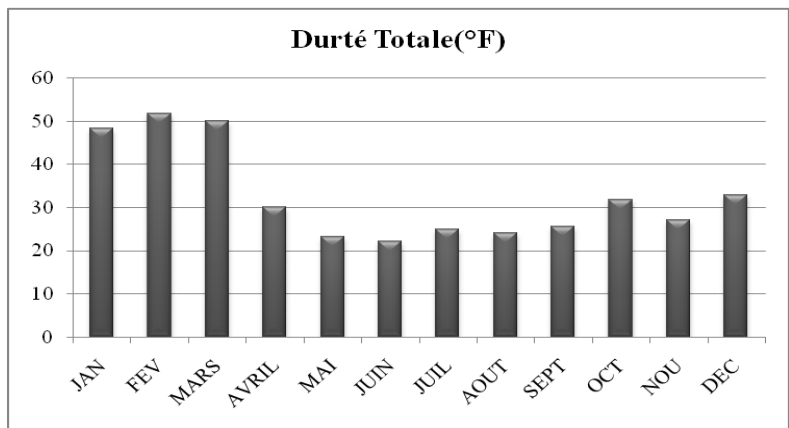

Figure 7 : Variation temporelle de la dureté totale.

\section{Calcium}

Les résultats enregistrés de la teneur en calcium durant la période d'étude représentent l'importance de cet élément dans les eaux du bassin versant du barrage Foum El-Khanga, avec des teneurs variables oscillent entre 119,44 mg/l (mois d'août) et 249,1 mg/l (mois de février) (Figure 8).

La diminution de la teneur en calcium durant la période sèche (mai à septembre, cela est expliqué selon Eugstre et hardie (1978 in Hacini et al., 2008) par la précipitation précoce des minéraux calcique, ou l'occurrence de la calcite.

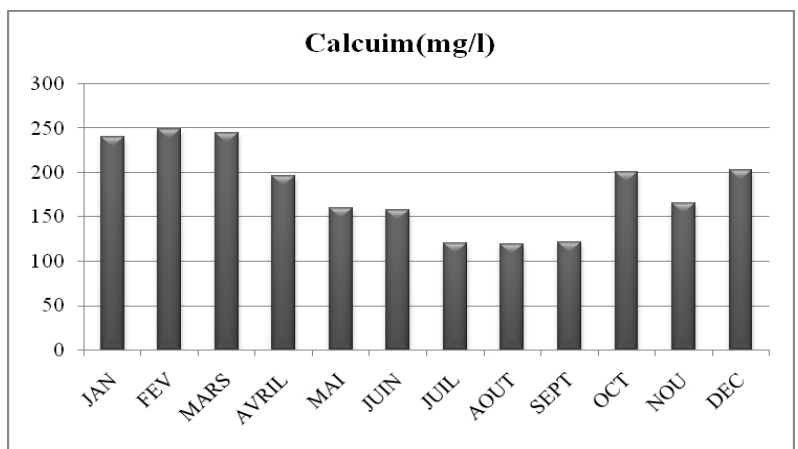

Figure 8 : Variation temporelle du calcium. 


\section{Magnésium}

Les valeurs enregistrées varient de $32,03 \mathrm{mg} / 1$ (mois d'avril) à 99,12 $\mathrm{mg} / \mathrm{l}$ (mois de février), avec une moyenne de 60,90 mg/l (Figure 9). Ces teneurs s'avèrent très hautement supérieures à ceux trouvés par Orou et al. (2016) dans les eaux souterraines du département d'Agboville (varié entre $0,064 \mathrm{meq} / 1$ au mois d'octobre et $0,76 \mathrm{meq} / 1$ au mois de mai).

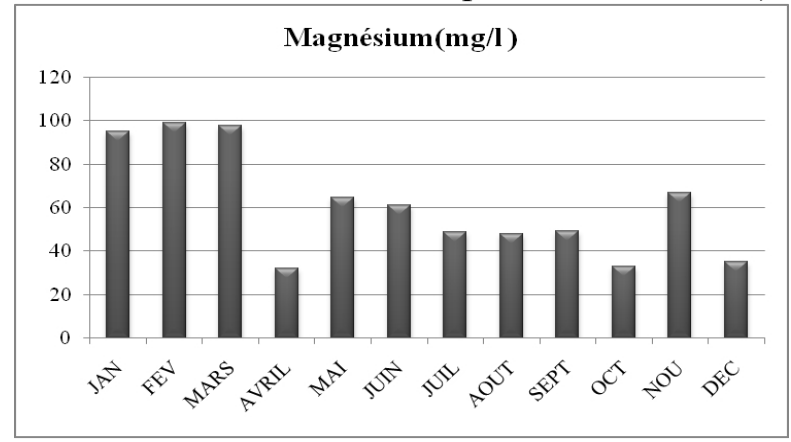

Figure 9 : Variation temporelle du magnésium.

\section{Titre alcalimétrique complet}

Le titre alcalimétrique complet mesure la teneur de l'eau en alcalin libre et en carbonate caustique. Le TAC fluctue au cours de notre étude de 4 $\mathrm{mg} / \mathrm{l}$ (mois de juillet) à 23,47 mg/l (mois d'avril) (Figure 10).

Les résultats de TAC de nos eaux sont très inférieurs à ceux rapportés par les travaux de Bentouati et Bouzidi (2011) des eaux souterraines de la Wilaya de Sétif (valeur minimale moyenne est de l'ordre de 28, 00 13,63 $\circ$ F).

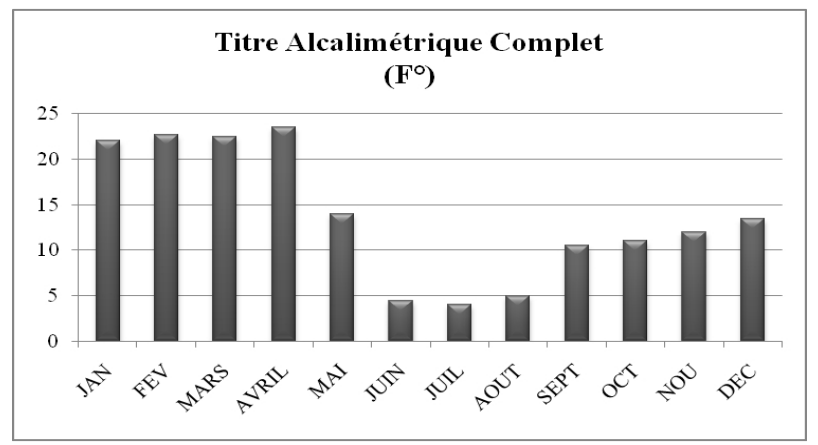

Figure 10 : Variation temporelle du titre alcalimétrique complet.

\section{Bicarbonate}

La valeur moyenne enregistrée au cours de la période d'étude en ion bicarbonate, est de l'ordre de $158 \mathrm{mg} / \mathrm{l}$, avec une valeur maximale de 276,94 $\mathrm{mg} / \mathrm{l}$ enregistrée au mois de février, et une minimale de $48,8 \mathrm{mg} / 1$ notée au mois de juillet (Figure 11). 
L'importance de la teneur du bicarbonate dans le barrage Foum ElKhanga surtout dans les saisons humides où on note l'augmentation du volume d'eau durant cette période, ce qui provoque une élévation de la teneur de bicarbonate dû à la dissolution des évaporites. Elle dû peut-être aussi à la nature des eaux résiduaires et à la composition des formations géologiques. Ces résultats sont largement supérieurs à ceux rapporté par Orou et al. (2016) dans les eaux souterraines du département d'Agboville (varié entre $0,02 \mathrm{meq} / 1$ dans la saison pluvieuse et $0,85 \mathrm{meq} / 1$ dans la saison sèche).

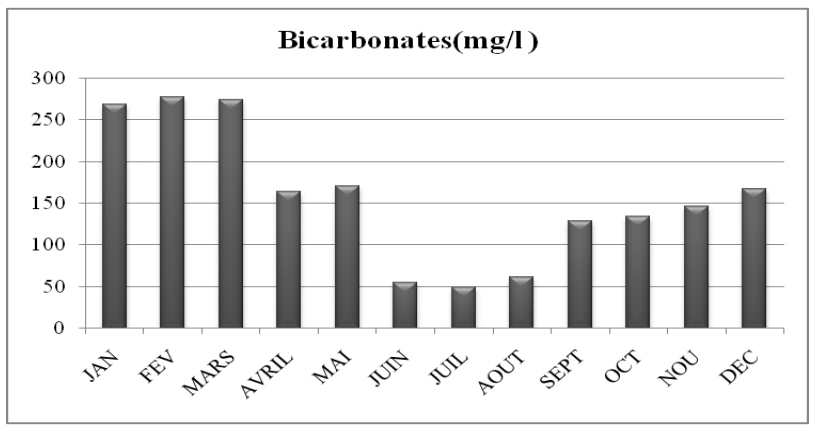

Figure 11 : Variation temporelle des bicarbonates.

\section{Chlorures}

Le suivi de l'évolution mensuel des chlorures dans le barrage de Foum El-Khanga permet de détecter d'importantes oscillations. En effet, les teneurs extrêmes maximale et minimale sont de $639,05 \mathrm{mg} / 1$ (mois de février) et $433,78 \mathrm{mg} / 1$ (mois d'août) respectivement, avec une valeur moyenne de 522,20 mg/l (Figure 12). Ces teneurs en chlorure restent supérieures à celles marquées par Mehanned et al., (2014) au niveau du barrage Sidi Chahed (valeurs moyennes oscille entre $248,86 \mathrm{mg} / 1$ et 263,57 $\mathrm{mg} / \mathrm{l})$.

Selon Halimi (2008), le pic de chlorures enregistré au niveau de ce barrage est dû à la géologie des terrains (lessivage du gypse et des argiles), la contamination agricole (pesticides) et les rejets domestiques.

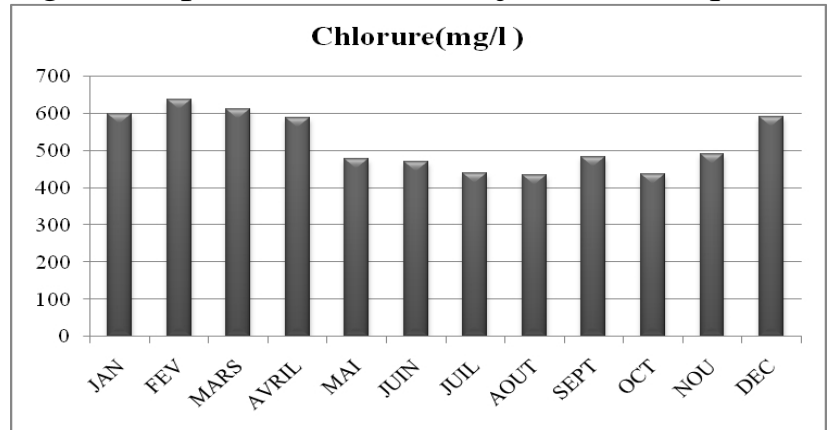

Figure 12 : Variation temporelle des chlorures. 


\section{Sulfate}

Dans le milieu d'étude, la valeur moyenne de sulfate est de l'ordre $325,85 \mathrm{mg} / \mathrm{l}$. La teneur du sulfate augmente en hiver $(428,78 \mathrm{mg} / \mathrm{l} \max$ en janvier) et diminue en été (139,5 mg/l min en juin) (Figure 13). L'évolution mensuelle des teneurs des sulfates montrent des concentrations plus élevées en période humide que celles en période sèche. Ces fortes teneurs peuvent être dues aux activités agricoles. Les teneurs en sulfate de nos eaux sont supérieures à celles enregistrées par Chahboun et al., (2013) au niveau du barrage Hassan II (valeur maximale est de l'ordre de 101,67 mg/ l).

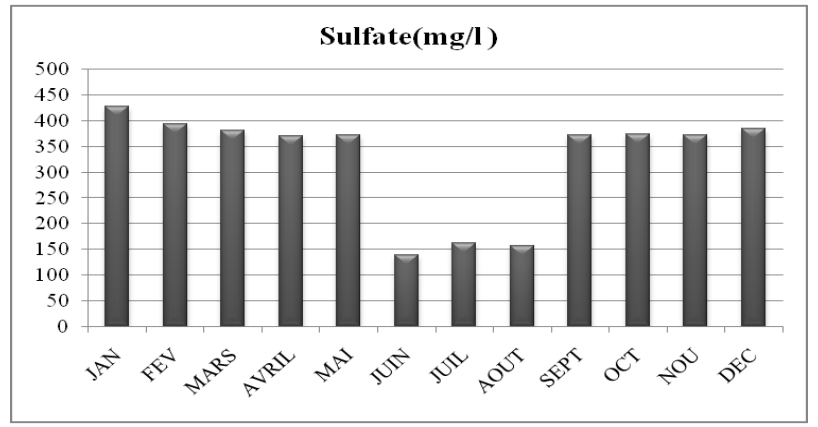

Figure 13 : Variation temporelle des sulfates.

\section{Nitrate}

Les nitrates sont utilisés comme indicateur de pollution. Ils jouent le rôle de fertilisant pour les plantes qui assimilent l'azote sous la forme $\mathrm{NO}_{3}$ (Djermakoye, 2005).

L'histogramme des teneurs en nitrates (Figure 14) montre une légère variation qui oscille entre $7 \mathrm{mg} / \mathrm{l}$ (au mois de juillet) et 15,4 mg/l (au mois de janvier).

L'augmentation des teneurs en nitrates dans les eaux pendant la saison pluvieuse par rapport à la saison sèche peut être due au lessivage des fertilisants utilisés dans les sols agricoles situés sur les bords du barrage. Ces résultats sont largement inférieurs à celles marquées par Bentouati et Bouzidi (2011) des eaux souterraines de la Wilaya de Sétif (valeur maximale

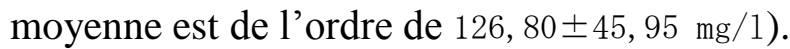

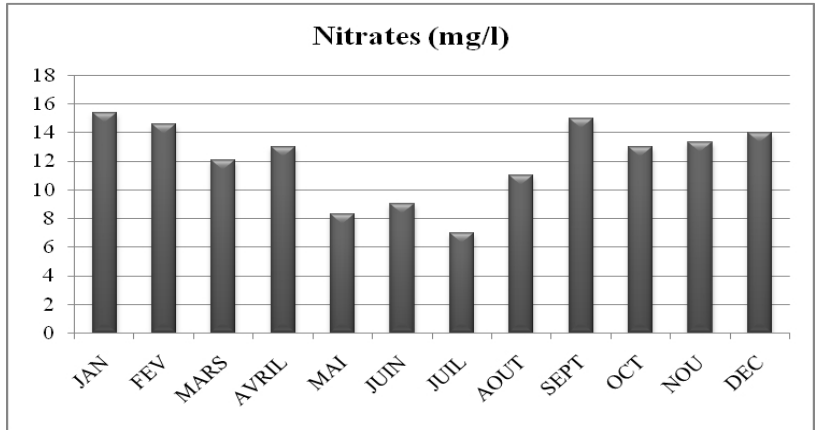

Figure 14 : Variation temporelle des nitrates. 


\section{Nitrite}

Les valeurs de ce paramètre dans les eaux étudiées sont très variables et oscillent entre $0,057 \mathrm{mg} / \mathrm{l}$ (au mois du juin) et $1,97 \mathrm{mg} / 1$ (au mois d'avril) (Figure 15). Nos teneurs en nitrite se rapprochent à ceux obtenus par Bentouati et Bouzidi (2011) des eaux souterraines de la Wilaya de Sétif (valeur maximale moyenne est de l'ordre de 1,261 $\pm 0,709 \mathrm{mg} / 1$ ).

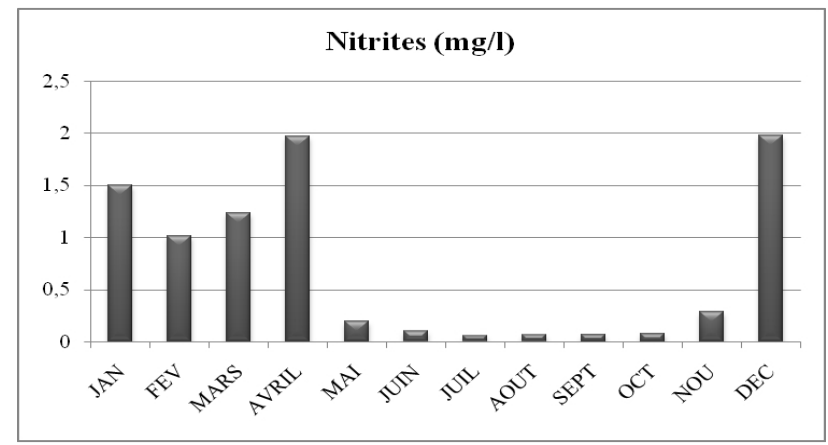

Figure 15 : Variation temporelle des nitrites.

\section{Ammonium}

L'histogramme des variations mensuelles des teneurs en ammonium (Figure 16) montre des fluctuations allant de $0,04 \mathrm{mg} / \mathrm{l}$ (au mois d'avril) à $0,57 \mathrm{mg} / \mathrm{l}$ (au mois de décembre).

La saison pluvieuse enregistre le taux le plus élevé en ammonium par apport à la saison sèche, ce qui traduit le processus de lessivage des terres agricoles entourant le barrage. Nos résultats sont comparables à ceux trouvés par Mehounou et al. (2016) au niveau des eaux souterraines et superficielles de la zone de production cotonnière d'Aplahoué (avec une valeur maximale de l'ordre de $1,5 \mathrm{mg} / \mathrm{l})$.

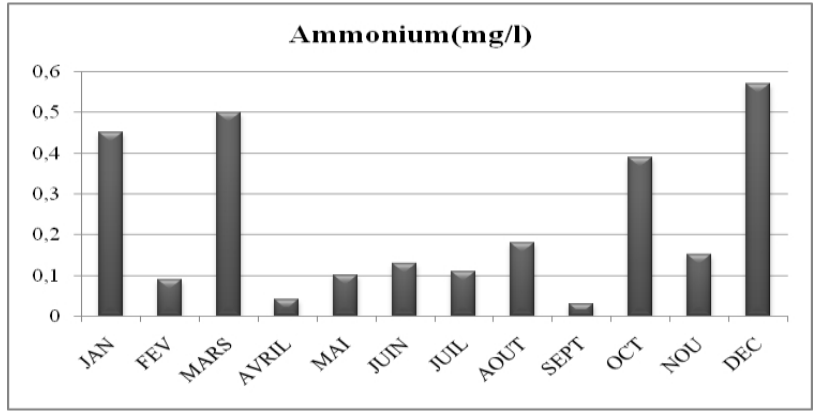

Figure 16 : Variation temporelle de l'ammonium.

\section{Phosphate}

Les teneurs en phosphates dans les eaux étudiées sont très faibles et parfois nulles, elles oscillent entre $0 \mathrm{mg} / \mathrm{l}$ et $0,13 \mathrm{mg} / \mathrm{l}$ (Figure 17), avec une tendance à l'augmentation en période humide. Selon Martin (1980), les 
agents atmosphériques, vent et pluie représentent des sources de phosphates importantes surtout quand le débit fluvial est faible. Nos résultats restent largement inferieurs par rapport à ceux signalés par Akatumbila et al., (2016) et Makhoukh et al., (2011) (valeurs maximales sont de l'ordre de 39,48 mg/l et $1,48 \mathrm{mg} / \mathrm{l}$ respectivement).

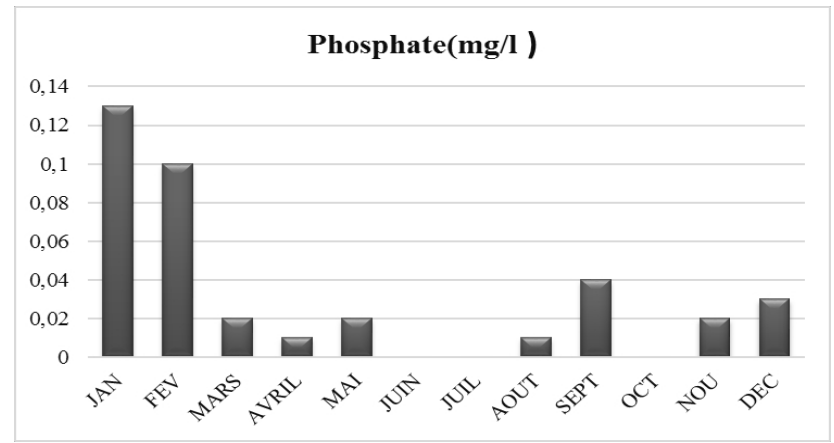

Figure 17 : Variation temporelle des phosphates.

\section{Fer}

La figure 18 montre, les variations de la concentration en ions de fer des eaux du barrage Foum El-Khanga. Ces teneurs sont très faibles, elles varient entre $0,01 \mathrm{mg} / \mathrm{l}$ (au mois de décembre) et $0,34 \mathrm{mg} / \mathrm{l}$ (au mois de février).

La présence du fer pourrait provenir de lessivage du sol agricole et du contexte géologique régional (richesse en ferromagnésiens dans les sédiments) (Kaimoussi, 1999).

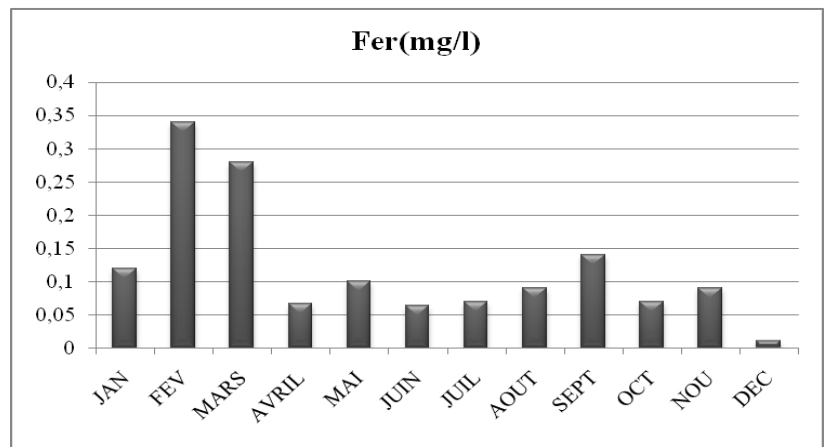

Figure 18 : Variation temporelle du fer.

\section{Demande biologique en oxygène}

Dans notre étude, les valeurs de la DBO varient entre $3 \mathrm{mg} / \mathrm{l}$ (au mois de mai) et $9 \mathrm{mg} / \mathrm{l}$ (au mois d'août), avec une moyenne de 5,33 mg/l (Figure 19).

L'augmentation des teneurs en DBO durant la période sèche (mois d'août) peut être expliquée par l'instauration des conditions de dégradation de la matière organique par les microorganismes, dont l'activité s'intensifie avec 
la diminution de la vitesse d'écoulement et avec le réchauffement des eaux. Cette activité, consommatrice d'oxygène, est à l'origine de l'autoépuration des eaux (Bremond et Perrodon, 1979). Cependant en période humide les eaux de pluie contribuent à la dilution de la charge organique.

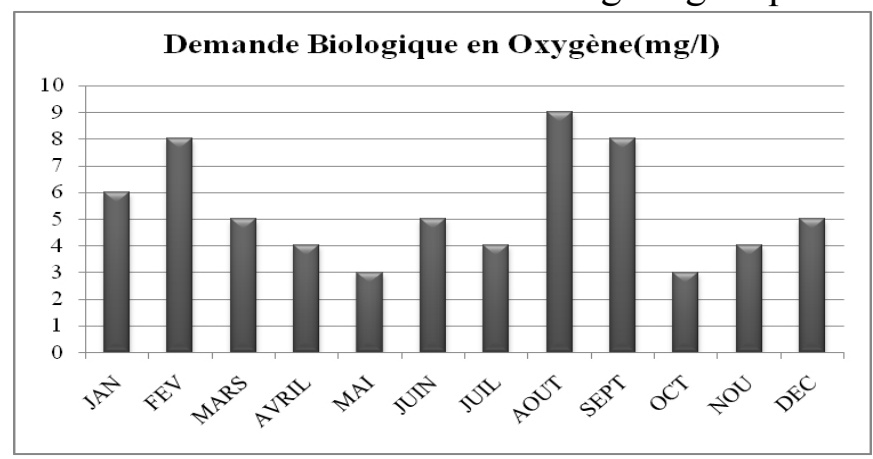

Figure 19 : Variation temporelle de la demande biologique en oxygène.

\section{Demande chimique en oxygène}

Les variations des teneurs en DCO, au cours de notre suivi sont représentées par la figure 20. Elles oscillent entre $35 \mathrm{mg} \mathrm{O}_{2} / 1$ (mois d'octobre) et $55 \mathrm{mg} \mathrm{O}_{2} / \mathrm{l}$ (mois de février) avec une moyenne de $47 \mathrm{mg} \mathrm{O}_{2} / \mathrm{l}$. Au niveau du barrage Foum El-Khanga, la DCO évolue dans le même sens que le gradient de minéralisation. Ceci a été relevé par Ezzaouaq (1991) dans l'estuaire de Bouregreg.

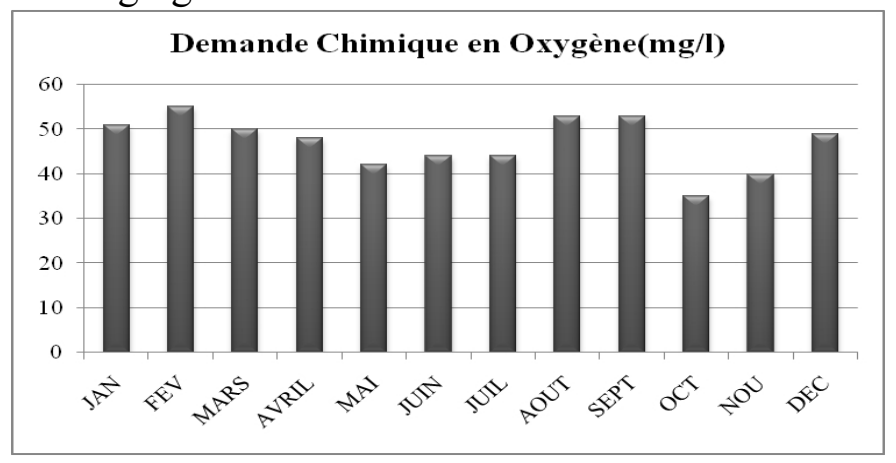

Figure 20 : Variation temporelle de la demande chimique en oxygène.

\section{Analyse en composante principale (ACP)}

Cette analyse a été réalisée sur un tableau de données centréesréduites (ACP normée) de 19 variables durant les 12 mois: Température de l'eau (Temp), pH, Oxygène dissous $\left(\mathrm{O}_{2}\right)$, Conductivité (Cond), Turbidité (Tur), Dureté totale (TH), Calcium $\left(\mathrm{Ca}^{2+}\right)$, Magnésium $\left(\mathrm{Mg}^{2+}\right)$, Titre alcalimétrique complet (TAC), Bicarbonates $\left(\mathrm{HCO}_{3}\right)$, Chlorures $(\mathrm{Cl})$, Nitrates $\left(\mathrm{NO}_{3}{ }^{-}\right)$, Nitrites $\left(\mathrm{NO}_{2}^{-}\right)$, Ammonium $\left(\mathrm{NH}_{4}{ }^{+}\right)$, Sulfates $\left(\mathrm{SO}_{4}{ }^{3-}\right)$, 
Phosphates $\left(\mathrm{PO}_{4}{ }^{2-}\right)$, le Fer $(\mathrm{Fe}+)$, Demande Biologique en Oxygène (DBO) et Demande chimique en Oxygène (DCO).

Les deux axes pris en considération pour décrire les corrélations entre les variables physico-chimiques et les mois de prélèvement, détiennent $66,31 \%$ de la variance avec respectivement $49,99 \%$ pour l'axe 1 et $16,32 \%$ pour l'axe 2 (Figure 21).

L'examen des données relatives à la matrice de corrélation entre les variables physico-chimiques et le nuage du point des 12 mois nous a permis de révéler que :

La température de l'eau est corrélée positivement avec $\mathrm{F} 1(\mathrm{r}=0,81)$. Ce dernier, par son pôle positif, regroupe les mois les plus chauds (juin, juillet, août et septembre).

Les paramètres suivants sont corrélées négativement avec F1: l'oxygène dissous $(\mathrm{r}=-0,92)$, le Chlorure $(\mathrm{r}=-0,91)$, le Calcium $(\mathrm{r}=-0,88)$, la turbidité $(\mathrm{r}=-0,84)$, le phosphate $(\mathrm{r}=-0,81)$, le titre alcalimétrique complet $(\mathrm{r}=-0,79)$, le sulfate $(\mathrm{r}=-0,77)$, le titre hydrométrique $(\mathrm{r}=-0,76)$, le nitrate $(\mathrm{r}=-0,74)$, le nitrite $(\mathrm{r}=-0,72)$, le bicarbonate $(\mathrm{r}=-0,68)$, le magnésium $(\mathrm{r}=-$ $0,63)$, le $\mathrm{pH}(\mathrm{r}=-0,56)$ et l'ammoniac $(\mathrm{r}=-0,50)$. Ce pôle regroupe donc, la majorité des paramètres qui déterminent le degré de minéralisation et de pollution de l'eau caractérisant les mois froids (novembre, décembre, janvier et février).

L'axe factoriel F2 se trouve bien corrélé avec la DBO $(\mathrm{r}=0,84)$ et la DCO $(r=0,67)$, ces deux paramètres sont situés du côté positif de la composante, et avec aussi la conductivité $(\mathrm{r}=-0,55)$ qui se situe dans le pôle négatif de cette composante.
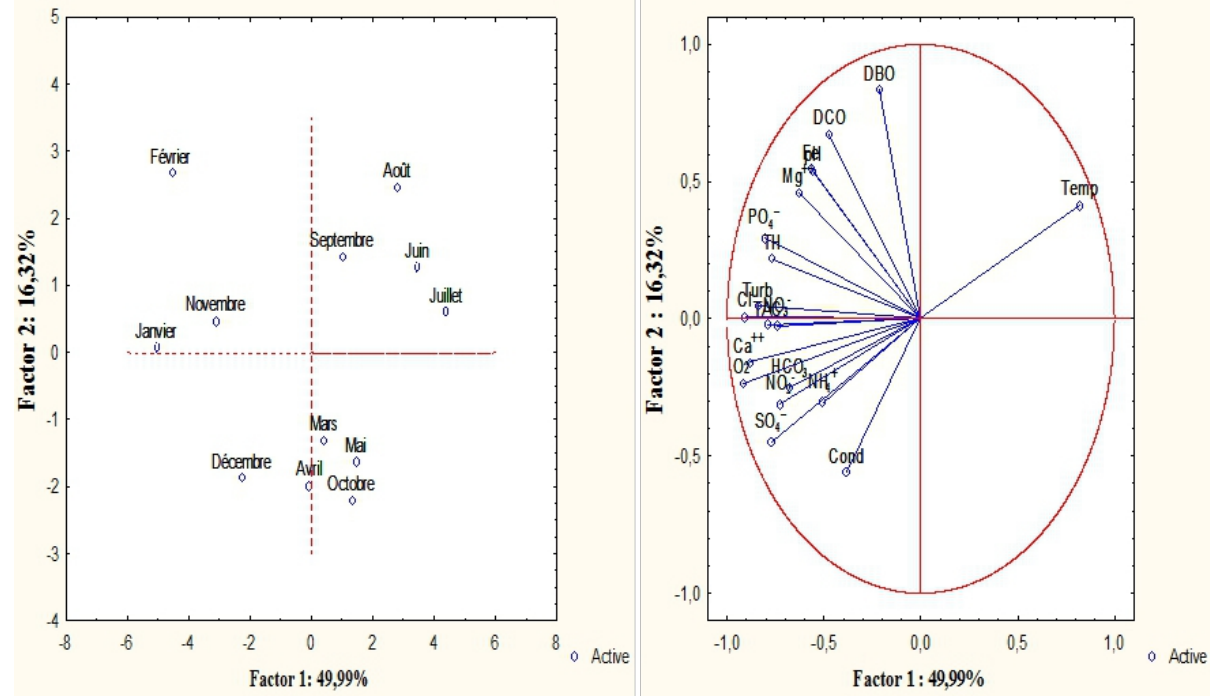

Figure 21 : Projection des variables physico-chimiques et des mois sur les plans factoriels (1X2). 
Nos résultats sont cohérents avec les conclusions de Jerônimo et al. (2014) qui ont montré que la saison froide est fortement corrélée avec la conductivité électrique, la dureté totale, le $\mathrm{pH}$, l'oxygène dissous et le fer. Alors que, la saison chaude est fortement corrélée avec l'ammonium, l'alcalinité et la température.

\section{Conclusion}

Les analyses de la qualité des eaux du barrage Foum El-Khanga révèlent une minéralisation importante de ces eaux vue les valeurs élevées de la conductivité électrique (au voisinage de $1900 \mu \mathrm{S} / \mathrm{Cm}$ ), des chlorures (522,20 mg/l), du calcium $(203,1 \mathrm{mg} / \mathrm{l})$ et du sulfate $(428,78 \mathrm{mg} / \mathrm{l})$. L'analyse en composante principale normée permet de dégager les corrélations existantes entre la saison sèche qui se caractérise par une élévation de la température, de l'ammonium et de l'alcalinité, alors que, durant la saison pluvieuse se sont les charges minérales et organiques qui sont les plus remarquables.

\section{References:}

1. Achour, S. (2001). Incidence des procédés de chloration, de floculation et d'adsorption sur l'évolution de composés organiques et minéraux des eaux naturelles. Thèse de Doctorat d'Etat en Sciences Hydrauliques. Université de Tizi-Ouzou, Algérie. 231 p.

2. AFNOR. (1985). Recueil des normes françaises des eaux, méthodes

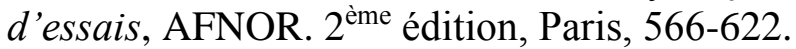

3. Ahonon, A. (2011). Evaluation De La Qualité Physico-chimique et Bactériologique Des Eaux De Surface Dans Les Zones Montagneuses Du Sud-Ouest Du Togo : Cas Du Canton De Lavie. Thèse De Master International En Environnement Eau et Santé. Université De Lome. $42 \mathrm{p}$.

4. Akatumbila, L., Mabiala, M., Lubini, A., Pwema, K., \& Musibono, E. A. (2016). Contribution à l'évaluation de la qualité physicochimique de l'eau : cas de la rivière urbaine Gombe de Kinshasa/République démocratique du Congo. Larhyss Journal, $n^{\circ} 26$, Juin 2016, pp. 7-29.

5. Bentouati, L., \& Bouzidi., A. (2011). Etude de la qualité des eaux souterraines de la Wilaya de Sétif » ScienceLib Editions Mersenne, 3 : 111-207.

6. Bouderka, N., Souid, A.K., Lakhili, F., Lahrach, A., \& Benabdelhadi, M. (2016). Evaluation De L'impact De La Pollution Agricole Sur La Qualite Des Eaux Souterraines De La Nappe Du Gharb. European Scientific Journal, 12 (11), 509-524. 
7. Bremond, R., \& Perrodon, C. (1979). Paramètres de la qualité des eaux. Ministère de l'environnement et cadre de vie. $2^{\text {ème }}$ ed : 259 p.

8. Chahboun, M., Chahlaoui, A., Zaid, A., \& Ben Moussa, A. (2013). Contribution à la caractérisation physicochimique des eaux du lac réservoir du barrage Hassan II. Larhyss Journal, $\mathrm{n}^{\circ}$ 14, pp. 61.

9. Chaouki, I., Mouhir, L., Fekhaoui, M., El Abidi, A., Bellaouchou, A., \& El Morhit, M. (2015). Application de l'analyse en composantes principales (ACP) pour l'évaluation de la qualité des eaux usées industrielles de Salam Gaz - Skhirat. J. Mater. Environ. Sci. 6 (2) 455-464.

10. Derwich, E., Benaabidate, L., Zian, A., Sadki, O., \& Belghity, D. (2010). Caractérisation physico-chimique des eaux de la nappe alluviale du Haut Sebou en aval de sa confluence avec Oued Fes. Larhyss Journal, $\mathrm{N}^{\circ}$ 08. 101-112.

11. Djermakoye, H. (2005). Les eaux résiduaires des tanneries et des teintureries ; Caractéristiques physico-chimiques, bactériologiques et impact sur les eaux de surface et les eaux souterraines. Thés Doc Pharmacie. Université de BAMAKO. 123p.

12. Ezzaouaq, M. (1991). Caractérisation hydrodynamique, physicochimique et bactériologique des eaux superficielles de l'estuaire du Bouregreg (Maroc) soumis aux rejets des villes de Rabat-Salé. Thèse D.E.S. Fac. Sci. Rabat, 140 p.

13. Ghazali, D., \& Zaid, A. (2012). Caractérisation physico-chimique des eaux de la source AIN SALAMA-JERRI (région de Meknes). Science Lib Editions Mersenne : Volume 4, N 120106, 13 p.

14. Hacini, M., Oelkers, E.H., \& Kherici, N. (2008). Le Comportement $\mathrm{du}$ lithium et du bore durant l'évaporation complète du Chott Merouane dans le Sahara septentrional Algérie. 10p.

15. Halimi, S. (2008). Ressources et essai de gestion intégrée des eaux du bassin versant d'oued Cherf/Sedrata (Nord - Est Algérien). Thèse de Magister, Université Badji Mokhtar Annaba, Faculté des sciences de la Terre Département de Géologie, Option : Hydrogéologie. 126p.

16. Harrat, N., \& Achour, S. (2010). Pollution physico-chimique des eaux de Barrage de la région d'El Tarf. Impact sur la chloration, Larhyss Journal, n8, 47-54.

17. Harrat, N., \& Achour, S. (2011). Qualité et réactivité des eaux de barrage de Zit El Amba alimentant la station de traitement d'Azzaba, Courrier du Savoir Scientifique et Technique, $\mathrm{n}^{\circ} 11,113-117$.

18. Hebert, S., \& Legre, S. (2000). Suivi de la qualité de l'eau des rivières et petits cours d'eau. Direction du suivi de l'état de l'environnement. Ministère de l'Environnement Gouvernement $d u$ Québec. 5 p. 
19. Jemali, A., \& Kefati, A. (2002). Réutilisation des eaux usées au Maroc. Forum sur la gestion de la demande en eau. Mars 2002, Direction du Développement et de la Gestion D’irrigation / Madref/ Rabat.

20. Jerônimo, G.T., Pádua, S.B., Bampi, D., Gonçalves, E.L.T., Garcia, P. Ishikawa, M.M., \& Martins, M.L. (2014). Haematological and histopathological analysis in South American fish Piaractus mesopotamicus parasitized by monogenean (Dactylogyridae). Braz. J. Biol, vol. 74, no. 4, p. 1000-1006.

21. Kahoul, M., \& Touhami M. (2014). Evaluation de la qualité physicochimique des eaux de consommation de la ville d'Annaba (Algérie). Larhyss Journal, $n^{\circ} 19$, pp. 129-138.

22. Kaimoussi, A. (1996). Etude de la variabilité de l'accumulation des métaux lourds dans les différents compartiments (sédiment, mollusques et algues) du littoral de la région d'El Jadida. Thèse de 3ème cycle, Université Chouaib Doukkali, Faculté de Science. El Jadida. 147 p.

23. Lakhili, F., Benabdelhadi, M., Bouderka, N., Lahrach, H., \& Lahrach, A. (2015). Etude de la qualité physicochimique et de la contamination métallique des eaux de surface du bassin versant de Beht (Maroc). European Scientific Journal, Eedition, 11(11), 132147.

24. Makhoukh, M., Sbaa, M., Berrahou, A., \& Van. Clooster, M. (2011). Contribution à l'étude physico-chimique des eaux superficielles de l'oued Moulouya (Maroc Oriental). Larhyss Journal, n 09, pp. 149169.

25. Martin, J.M., (1980). Cycle des éléments chimiques dans les estuaires. Océanis 5 (fasc. Hors série) : $517-520$.

26. Mehanned, S., Chahlaoui, A., Zaid, A., Samih, M., \& Chahboune, M. (2014). Typologie de la qualité physico-chimique de l'eau du barrage Sidi Chahed Maroc. J. Mater. Environ. Sci. 5 (5) 1633-1642.

27. Mehounou, J.P., Josse, R.G., Dossou-Yovo, P., Senou, S.F., \& Toklo, R.M. (2016). Caractérisation physico-chimique et microbiologique des eaux souterraines et superficielles dans la zone de production cotonnière d'Aplahoué. J. Appl. Biosci. 103 :9841 - 9853.

28. Orou, R.K., Soro, G., Soro, D.T., Fossou, R.M.N., Onetie, O.Z., Ahoussi, E.K., \& Soro, N. (2016). Variation Saisonnière De La Qualité Physico-Chimique Des Eaux Souterraines Des Aquifères d'Altérites Du Département d'Agboville (Sud-Est De La Côte d'Ivoire). European Scientific Journal, 12 (17),81-100.

29. Ouahchia, C., Hamaidi-Chergui, F., Hamaidi, M.S., \& Saidi, F. (2015). Qualité bactériologique de l'eau potable des différents 
réservoirs et chez les consommateurs de la commune de Tipaza alimentes par la station de Sidi Amar à partir de l'eau de surface du lac barrage de Boukourdane. Larhyss Journal, n²3, 139-154.

30. Rodier, J. (1984). Analyse de l'eau, eaux naturelles, eaux résiduaires, eau de mer. $7^{\mathrm{ème}}$ édition, DUNOD Paris. $1365 \mathrm{p}$.

31. Rodier, J., Legube, B., Merlet N., et al. (2009). L'analyse de l'eau. $9^{\text {ème }}$ édition, DUNOD Paris, p. 1217, 1242, 1293, 1294, 1332. 